\title{
Look What I'm Interested in! Toward a Better Understanding of How Personalization and Self-Reference Drive News Sharing
}

\author{
Verena Thürmel \\ LMU Munich \\ thuermel@bwl.lmu.de
}

\author{
Benedikt Berger \\ LMU Munich \\ benedikt.berger@bwl.lmu.de
}

\author{
Thomas Hess \\ LMU Munich \\ thess@bwl.lmu.de
}

\begin{abstract}
Along with the continuing shift from traditional to digital news consumption, many news consumers share news via social networks and messaging services. Hence, news providers benefit from an increase in user involvement and a growing awareness of their news offerings. Although personalizing digital news offerings has become common practice, we know little about how personalization affects news sharing. Building on the stimulus-organism-response model, we propose a comprehensive framework to investigate how personalization and self-referential cues impact users' sharing intention mediated by their cognitive and affective reactions. To test our research model, we conduct an experiment with a fictitious news application and analyze the results using partial least squares structural equation modeling. The results reveal that personalization and self-reference impact users' perceived preference fit and perceived enjoyment, which in turn drive news sharing. The findings have important implications for researchers and news providers.
\end{abstract}

\section{Introduction}

The expansion of ubiquitous internet access, accelerating connection speed rates, and the increasing use of mobile devices have tremendously changed today's media landscape, resulting in a progressive shift from traditional to digital news consumption. More than a third of US news consumers currently use online channels as their main news source [1]. A key feature of online media channels is their interactivity [2] since users do not merely passively consume online news, i.e. by reading articles, but also actively engage with the news content, i.e. by commenting or sharing articles. Sharing news content via social networks or messaging services has become common behavior among news consumers [1]. These social referrals are a valuable marketing tool for many news providers, since they do not only increase the involvement of the person sharing a news article but also draw the recipient's attention to the news offering [2]. Hence, in the highly competitive digital landscape, news providers can no longer solely rely on offering informative and compelling news content but must also provide an engaging news offering that encourages users to share articles [2].

Numerous studies have investigated which content, network, and user characteristics influence the dissemination of online news. For example, content with a positive valence or high informational value is more likely to be shared than a negative or neutral content $[3,4]$. Moreover, prior research has investigated which underlying network structures of social media platforms [5, 6] and user characteristics [7] drive news sharing. Kümpel et al. [2] suggest the consideration of ongoing, contemporary technological advances in the digital media environment when investigating news sharing. An important advancement resulting from news providers' increasing ability to collect and analyze user data is the possibility to offer personalized news offerings, i.e. news offerings that are tailored to users' individual interests and preferences [8]. Several studies have shown that personalization evokes favorable user responses, such as customer satisfaction [9] and a greater willingness to pay [10]. However, to the best of our knowledge no study has investigated how personalization impacts users' sharing behavior. Therefore, we draw on the stimulus-organism-response model to investigate whether personalized news offerings enhance users' sharing of news. Additionally, we examine how self-referential cues moderate and users' cognitive and affective reactions mediate this relationship. Therefore, we pose the following research question:

How do personalization and self-reference in digital news offerings impact users' intention to share news articles?

To answer this question, we conducted a vignettebased online experiment, in which we confronted participants with a fictitious news application. Our 
findings provide valuable theoretical and practical implications by showing that personalization and selfreferential cues impact users' perceived preference fit and perceived enjoyment, which in turn affect their sharing intention.

The remainder of this paper is structured as follows: First, we draw on recent literature to elaborate on the theoretical foundation. We then present our research model and develop the hypotheses. After that, we outline our research method before analyzing and discussing the results. Third, we derive theoretical and practical implications and conclude with recommendations for further research.

\section{Theoretical foundation}

\subsection{Personalization and self-reference}

The shift from traditional to digital news consumption lowers boundaries for the production and distribution of news content and enables media companies to provide large amounts of news content to their customers in a timely and cost-efficient manner [11]. As a result, customers can choose between a plethora of news content alternatives. To reduce this information overload [12], media companies use personalization systems to provide users with personalized news offerings that correspond to their individual interests and preferences [8].

Personalization can be defined as the ability to provide customers with tailored offerings based on knowledge about their interests and preferences [8]. In the news context, personalization aims to "deliver the right content to the right person at the right time to maximize immediate and future business opportunities" (p. 867) [13]. This can be achieved by adapting the content, presentation format, or timing of news offerings to customers' individual needs [13]. According to Adomavicius and Tuzhilin [8], the personalization process consists of three stages, namely understanding the customer, delivering a personalized offering based on the knowledge about the customer, and measuring the personalization impact, e.g. customer satisfaction. First, to understand the customer, personalization systems collect data by explicitly asking users about their preferences or implicitly tracking their behavior to infer preferences, e.g. based on their purchasing history. Next, personalized offerings are generated based on different recommendation-based matchmaking techniques [8]. Content-based recommendation algorithms generate offerings based on items similar to users' past preferences, whereas collaborative-based recommendation algorithms choose items that are most liked by other users with similar taste and preferences. Lastly, the personalization system assesses the success of the personalization effort in order to adapt the personalization strategy accordingly. Investigating the influence of personalized media offerings on users' behavioral response has been the focus of many studies in the fields of information systems, marketing, and human-computer interaction. These studies show that web personalization leads to positive business outcomes such as a higher assessment of website value in terms of website stickiness and willingness to pay [e.g. 10], as well as increased customer satisfaction [e.g. 9].

However, $\mathrm{Li}$ [14] argues that favorable personalization outcomes depend on users' perception of personalized offerings as they do not necessarily perceive personalized offerings as personalized. In a study on advertising messages, Li found that favorable personalization effects occur only when users perceive a message as personalized, regardless of whether or not it is actually personalized. Likewise, Kramer [15] found that users are more likely to rely on personalized recommendations when they recognize that the recommendations are tailored to their expressed preferences. Accordingly, Choi et al. [16] found that personalized recommender systems lead to a higher level of customer satisfaction and purchase intention when self-referential cues inform users that the recommendation was personalized based on their individual preferences. These findings indicate that using self-referential hints when presenting personalized offerings can strengthen the positive effect of personalization. Tam and Ho [13] define selfreference as the association of web stimuli with the self, which is achieved by including cues such as the user's name or explicit tags such as "unique recommendations to you" that activate concepts stored in users' selfschemes. Psychological research has shown that the processing of self-referential information is distinct from the processing of objective information [17]. The human brain processes information that is associated with the self differently than non-self-referential information, resulting in deeper and more extensive information processing [18], as well as in a greater elaboration of a product's benefits [19]. Thus, selfreferential stimuli attract more attention and elicit higher click-rates and evaluations of the recommended items [13]. Consequently, the positive outcomes of personalization might be reinforced by supplementing personalized news offerings with self-referential cues.

\subsection{News sharing}

In recent years, news sharing via social media has become a fundamental part of online news distribution 
and consumption [2]. Sharing buttons provided on news websites and applications serve as a convenient tool to share news content with others via social networking sites such as Facebook and Twitter, or messaging services such as WhatsApp and Facebook Messenger. By adapting the definition of Kümpel et al. [2], we refer to news sharing as the "practice of giving a defined set of people access to news content" (p. 2), which comprises posting referrals on social networking sites as well as sharing news content via messaging services, with the goal to recommend news articles to others.

Scholars from various fields have investigated which content, network, and user characteristics impact the dissemination of online news. To understand the dissemination of content, IS research primarily focuses on investigating the underlying network structures of social media. For example, Susarla et al. [5] investigated the dissemination of word of mouth in social networks, showing that its spread depends on the responsiveness of recipient ties. Moreover, Yoo et al. [6] revealed that a cascade, i.e. the diffusion of given content, is affected by the spread of similar content.

Apart from the network structure, user characteristics are another aspect that influences the online sharing of news articles. Previous research has shown that the main motivations that drive news sharing are self-serving, altruistic, and social motives. First, users share news content for self-serving motives, such as shaping the impression others have of them by creating a positive self-image and signaling one's individual identity [20]. Furthermore, as an altruistic gesture, users share content on social networks to disseminate information that might be useful for or interesting to others [21]. Lastly, news content is shared to strengthen social relationships [7].

Finally, content-related factors play an important role in understanding the successful dissemination of news content. Previous studies have shown that in the context of news sharing, both cognitive and affective content characteristics impact news sharing. Berger and
Milkman [3] showed that the emotional component of online news is important when it comes to news sharing as emotionally arousing content is more likely to be shared. Likewise, Stieglitz and Dang-Xuan [4] revealed that sentiment positively impacts retweet quantity and retweet speed on Twitter. Moreover, cognitively appealing content has a higher probability of being shared as users, due to their confirmation bias, tend to share content that they are familiar with [22]. The confirmation bias theory states that people tend to share content which supports their current beliefs [23]. This biased sharing behavior underlies the mechanism of homophily, which is the tendency to associate with peers that are similar to oneself in terms of their characteristics and their environment $[22,24]$.

\section{Hypotheses development}

To develop our research model, we draw on the stimulus-organism-response (S-O-R) framework [25] which proposes that various environmental stimuli affect individuals' cognitive and affective states of processing, which in turn determine their behavioral response. Stimuli refer to cues from the external environment that attract consumers' attention. In the online environment, web content can be considered as a mix of stimuli that users experience when interacting with a website or mobile application. Web stimuli can take various forms, such as content and design features [10], or transparency features [26]. Furthermore, the organism represents users' cognitive and affective intervening process, which explains how a stimulus elicits a certain behavioral response. This intervening process reflects how users consciously and unconsciously process the incoming stimulus [27]. Lastly, the response represents users' final behavioral reaction which can be both internal (e.g. attitude or behavioral intention) as well as external (e.g. actual buying behavior) $[10,27]$.

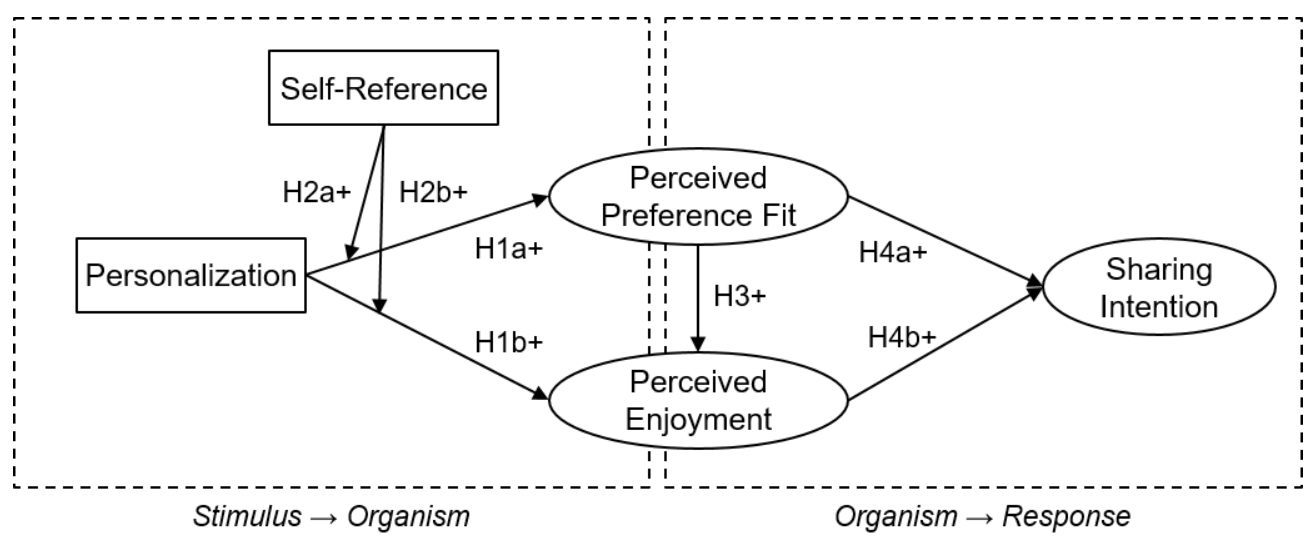

Figure 1. Research model 
Building on the S-O-R framework, our research model consists of two stages. First, we predict that personalization and self-reference, used as stimuli inherent in the digital news offering, impact users' cognitive and affective reactions toward the news offering. Second, we assume that users' cognitive and affective perceptions of the news offering impact their sharing intention. The resulting research model is depicted in Figure 1.

In the context of personalization, IS literature most frequently relies on users' perceived preference fit to assess their cognitive reaction $[10,26]$. Benlian [10] defines the perceived preference fit as "users' subjective evaluation of the extent to which a website's offerings correspond to their current preferences" (p. 231). Thus, to model users' cognitive reaction, we draw on the perceived preference fit. Since personalization systems aim to provide each user with the right content at the right time [13], we assume that the constant matching of users' needs with the provided content results in users experiencing a greater overlap between their preferences and the personalized news offering compared to standardized news offerings. Accordingly, consistent with prior IS research [10], we hypothesize that the personalization of the news offering elicits an increased perception of preference fit.

Hla: Personalization positively influences perceived preference fit.

To represent users' affective state, we follow previous IS studies $[10,26]$ by drawing on the construct of perceived enjoyment. Perceived enjoyment provides an affective measure of users' perception of whether interacting with a system is enjoyable and fun [28]. Previous IS studies have shown that users enjoy interacting with technical systems that respond to their actions [10, 26, 29]. The enhanced responsiveness of a system stimulates their sensory experience and subsequently elicits positive affective reactions [26, 29]. Since personalization cues respond to users' individual needs by adapting the displayed content accordingly, we hypothesize that personalization cues inherent in the digital news offering positively impact users' perceived enjoyment.

H1b: Personalization positively influences perceived enjoyment.

Several studies on personalization propose that users are more likely to rely on personalized offerings when they recognize that the offering was tailored to their preferences and they actually perceive it as personalized $[14,15]$. Consequently, we suggest that self-referential cues underlining the personalization effort enhance users' cognitive and affective reactions to personalized news offerings.

According to the customized label effect, Simonson [30] proposes that self-referential labels positively affect the perceived fit of personalized offerings. Hence, when evaluating a news offering labeled as being based on their individual preferences, users may perceive that the "personalized offering" indeed fits their preference as the self-referential cue attracts their attention and triggers deeper information processing $[13,18]$. This deeper information processing might lead to an increased perceived fit between own preferences and the personalized news offering.

H2a: Self-reference enhances the positive influence of personalization on perceived preference fit.

Prior research has indicated that users enjoy using interfaces that interactively respond to their preferences [26]. Hence, to enjoy the benefits of a personalized news offering, it is imperative that users actually become aware that the news offering is based on a personalization system that responds to their personal preferences. Thus, news providers can highlight the interactive character of their news offering by presenting self-referential cues to users. Therefore, we hypothesize that including self-referential cues in the personalized news offering enhances users' perceived enjoyment.

$H 2 b$ : Self-reference enhances the positive influence of personalization on perceived enjoyment.

Previous research has shown that the goodness of fit between a technological system's information representation and the task a user wants to fulfil can increase the enjoyment derived from interacting with the system $[28,31]$. The more relevant users perceive the represented information, the more they enjoy using the system due to their increased interest [28]. In the case of online news, a news offering consisting of articles that match users' individual preferences minimizes the information overload [12]. This supports users in finding suitable news content and leads to positive emotional reactions. Accordingly, we hypothesize that if users perceive that a news offering matches their preferences, they experience the offering as being more enjoyable and fun.

H3: Perceived preference fit positively influences perceived enjoyment.

When users decide which content to share, they are often driven by a phenomenon called confirmation bias [22]. Accordingly, they tend to primarily share content which supports their current beliefs [23]. This biased behavior can be explained by homophily, in that people tend to connect with others who are interested in similar topics $[22,24]$. Hence, we hypothesize that users are more likely to share content when it matches their interests and preferences.

H4a: Perceived preference fit positively influences the intention to share news.

Several studies have shown that users' sharing of news content depends on their internal emotional state. 
Stieglitz and Dang-Xuan [4] found that emotions drive the diffusion of information in social networks. Likewise, Berger and Milkman [3] indicated that content is more likely to be shared when it elicits positive emotions. Since content is often shared to create a positive self-image [21], sharing amusing content might help users to signal their sense of positivity. In line with these findings, we argue that news offerings that elicit positive emotional reactions among users, such as enjoyment and fun, enhance users' intention to share news articles.

H4b: Perceived enjoyment positively influences the intention to share news.

\section{Method}

\subsection{Experimental design}

To test the derived hypotheses, we conducted a vignette-based online experiment introducing a mobile news application using Qualtrics. We employed a 2 (no personalization vs. personalization) $\times 2$ (no selfreference vs. self-reference) between-subject design. As previous studies have shown, scenario induced vignettes elicit similar reactions to experiments in field settings [32]. Especially in online settings, vignette-based scenarios are commonly used to investigate consumer behavior [e.g. 26, 33].

After a short introduction to the study, we asked respondents to indicate their preferences for online news by choosing one of the following topics: economy, technology, or lifestyle. These topics are among the top categories of most news websites (e.g. The New York Times [34], BBC News [35]). Next, we randomly assigned the participants to one of the four experimental groups and introduced them to their vignette scenario.
In all four groups, we asked the participants to imagine using the fictitious mobile news application 'News Today'. Each group was presented with a distinct starting page of the news application that included four articles. The two groups in the self-reference domain (1a and $1 \mathrm{~b}$ ) were shown a starting page with the headline "Your personal starting page based on your interests". The other two groups ( $2 \mathrm{a}$ and $2 \mathrm{~b}$ ) were presented with a non-self-referential starting page with the headline "The top news". In addition, the two groups in the personalization domain (1a and $2 \mathrm{a}$ ) were presented with a personalized news offering. This offering comprised three articles matching their previously indicated, preferred topic and an article on another non-related topic, i.e. nature, which we did not ask for during the preference elicitation. The other two groups ( $1 \mathrm{~b}$ and $2 \mathrm{~b}$ ) were shown a single article on each of the topics economy, technology, and lifestyle, as well as an article on the topic nature. We then invited the participants to explore the news offering of 'News Today' and to read two of the four presented articles. All shown articles were condensed versions of recent articles from German news publishers. After reading an article, participants had to rate - on an eleven-point scale adapted from the net promoter score [36] - how likely it is that they would share the article to recommend it to someone. After reading two articles, we asked the participants to complete the post-experimental survey in the following order: dependent variables, manipulation check, and demographics. The described experimental procedure is depicted in Figure 2.

To verify whether self-reference was perceived as intended, we followed Tam and Ho [13] by asking participants whether they recall being shown a selfreferential headline on the starting page. Further, to test the treatment effect of personalization, participants had to rate on a seven-point Likert scale whether they

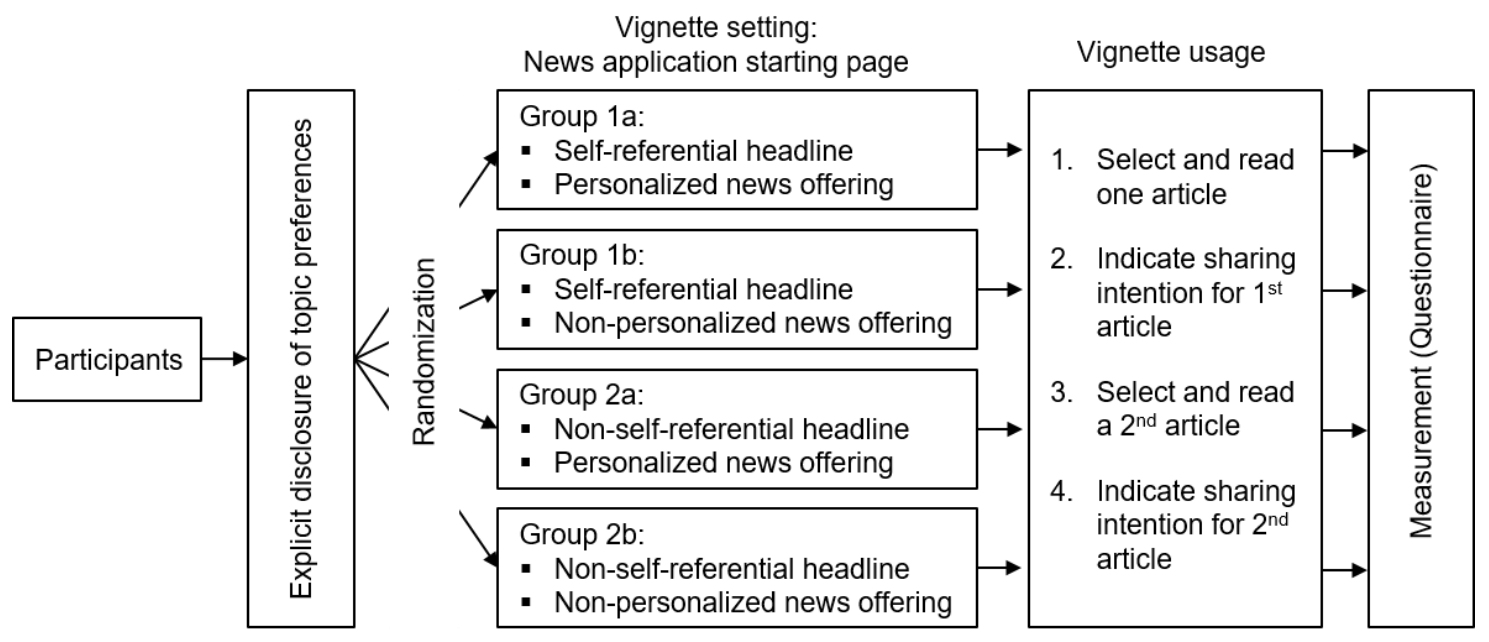

Figure 2. Experimental setup 
perceived the news offering to be based on their initially indicated preferred topic. Two independent samples ttests revealed significant group differences for selfreference and personalization on a one percent significance level.

\subsection{Operationalization of constructs}

To ensure that all constructs were measured in accordance with our definitions, previously established scales from the literature were carefully adapted to our study's context. To measure perceived preference fit and perceived enjoyment, we applied items from Benlian [10]. Further, sharing intention was assessed by adapting the 0 to 10 rating scale of the net promoter score by Reichheld [36], asking participants for both articles they read: "How likely is it that you would share the link to the article in a messaging service to recommend the article to someone?" as well as "How likely is it that you would post the link to the article on a social network to recommend the article to your followers?". In accordance with our definition of news sharing, these questions cover both one-to-one sharing via messaging services and the sharing of links in social networks. The resulting four items were averaged to model sharing intention [37]. Whereas the items of perceived preference fit were rated on seven-point Likert-type scales anchored at 1 (strongly disagree) and 7 (strongly agree), perceived enjoyment was measured by seven-point semantic differential scales. As the study was conducted in German, a back-translation assured that the meaning of the items was not altered through translation.

\subsection{Sample and data collection}

Before commencing with the data collection, a pretest was carried out to ensure the questionnaire's comprehensiveness and to verify that the manipulated vignettes were perceived as intended. Based on the feedback of 13 respondents, including IS researchers, practitioners, and students, the vignettes and the wording of the questionnaire were revised. The main study was conducted in Q2 2020. We distributed the questionnaire via the mailing list of a large public German university, as well as by posting it in student groups on social networks. Samples primarily composed of students are often used in related studies [e.g. 13, 26]. As an incentive to participate, we announced to raffle five vouchers worth $20 €$ each among all participants.

A total of 194 participants completed the questionnaire. $71.1 \%$ of the participants were female, the average age was 27.2 years, and $68.6 \%$ were students. Furthermore, $84.5 \%$ of respondents indicated that they use online news services several times a week. The final sample consisted of 48 participants in group 1a (personalization and self-reference), 46 participants in group $1 b$ (no personalization and self-reference), 51 participants in group $2 \mathrm{a}$ (personalization and no selfreference) and 49 participants in group $2 \mathrm{~b}$ (no personalization and no self-reference), resulting in four experimental groups of roughly equal sizes.

\section{Table 1. Results of confirmatory factor analysis}

\begin{tabular}{|c|c|c|c|c|c|c|c|c|}
\hline Construct & Items & $\begin{array}{c}\text { Factor } \\
\text { loadings }\end{array}$ & $\mathbf{C A}$ & CR & AVE & PPF & $\mathbf{P E}$ & SI \\
\hline \multirow{3}{*}{$\begin{array}{l}\text { Perceived } \\
\text { preference } \\
\text { fit (PPF) }\end{array}$} & $\begin{array}{l}\text { I like the way my preferences were matched on } \\
\text { News Today. }\end{array}$ & .896 & \multirow{3}{*}{.916} & \multirow{3}{*}{.947} & \multirow{3}{*}{.857} & \multirow{3}{*}{926} & \multirow{3}{*}{ 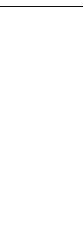 } & \multirow{3}{*}{ 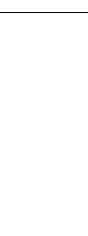 } \\
\hline & $\begin{array}{l}\text { The displayed selection of articles on News } \\
\text { Today comes close to my personal preferences. }\end{array}$ & .945 & & & & & & \\
\hline & $\begin{array}{l}\text { The articles provided on News Today coincide } \\
\text { with my preferences. }\end{array}$ & .936 & & & & & & \\
\hline \multirow{3}{*}{$\begin{array}{l}\text { Perceived } \\
\text { enjoyment } \\
(\mathrm{PE})\end{array}$} & $\begin{array}{l}\text { Reading articles on News Today was } \\
\text { (un)exciting. }\end{array}$ & .863 & \multirow{3}{*}{.859} & \multirow{3}{*}{.914} & \multirow{3}{*}{.781} & \multirow{3}{*}{.685} & \multirow{3}{*}{.884} & \\
\hline & $\begin{array}{l}\text { Reading articles on News Today was } \\
\text { (un)appealing. }\end{array}$ & .894 & & & & & & \\
\hline & Reading articles on News Today was (not) fun. & .894 & & & & & & \\
\hline $\begin{array}{l}\text { Sharing } \\
\text { intention (SI) }\end{array}$ & $\begin{array}{l}\text { How likely is it that you would share/post the } \\
\text { link to the article in a messaging service/on a } \\
\text { social network to recommend the article to } \\
\text { someone/your followers? }\end{array}$ & \multicolumn{4}{|c|}{$\begin{array}{l}\text { single item based on } \\
\text { summated scale }\end{array}$} & .365 & .364 & 1.000 \\
\hline
\end{tabular}




\section{Results}

\subsection{Measurement model analysis}

We conducted a confirmatory factor analysis using SmartPLS 3 [38] to assess the reflective measurement models. First, we used Cronbach's Alpha (CA) and composite reliability (CR) to evaluate the internal consistency reliability. Both exceeded the threshold value of 0.7 for all constructs, which can thus be regarded as reliable. Next, assessing indicator reliability revealed that all items had satisfactory factor loadings greater than 0.7 . To determine the convergent validity of our constructs, we assessed the average variance extracted (AVE). All constructs showed satisfactory values above the threshold of 0.50 . Further, discriminant validity on construct level was tested by assessing the Fornell-Larcker criterion, indicator cross loadings, and the heterotrait-monotrait ratio of correlations. The Fornell-Larcker criterion showed satisfactory values, all factor loadings exceeded their cross-loadings and all heterotrait-monotrait ratio values were below the threshold of 0.85 . Table 1 indicates the respective values for all constructs.

\subsection{Structural model analysis}

In order to obtain estimation results, we set up a partial least squares structural equation model (PLSSEM) in SmartPLS 3 [38]. To check for collinearity issues in the structural model, we examined the variance inflation factors (VIF) of all sets of predictor constructs. As all VIF values were below the critical threshold of 5, collinearity is not an issue [39]. To test significance of the path coefficients in our structural model, we conducted a bootstrapping procedure with 5,000 subsamples. To model the interaction effect of the moderating variable self-reference, we relied on the product indicator approach [39]. Figure 3 depicts the results of relationship evaluation and significance testing.

An assessment of the model's predictive power showed that the model explained $10.2 \%$ of variance in perceived preference fit, $49.7 \%$ of variance in perceived enjoyment, and $15.8 \%$ of variance in users' sharing intention. Consistent with H1a, the personalized news offering positively influenced respondents' perceived preference fit $(\mathrm{p}=.000)$. Further, consistent with H2a, we found a significant moderating effect of self-reference on the relationship between personalization and perceived preference fit $(p=.050)$, indicating that the effect of personalization on perceived preference fit is stronger when the news offering contains selfreferential cues. In contrast to $\mathrm{H} 1 \mathrm{~b}$, personalization has a negative direct effect on perceived enjoyment $(p=.020)$. A post-hoc mediation analysis [40] provided further insight into the relationship between personalization and perceived enjoyment by revealing a competitive mediation. Hence, in addition to the negative direct effect, personalization had a significant positive indirect effect on perceived enjoyment with perceived preference fit as mediator $(p=.000)$. Without controlling for mediation, the total effect of personalization on perceived enjoyment was not significant $(\mathrm{p}=.406)$, indicating that the direct and indirect effects with opposite signs cancelled each other out. Furthermore, self-reference had a significant moderating effect on the relationship between personalization and perceived enjoyment $(p=.041)$. However, in contrast to H2b, self-referential cues decrease the negative direct effect of personalization on perceived enjoyment. Consistent with $\mathrm{H} 3$, the hypothesized positive effect of perceived preference fit on perceived enjoyment was found to be strong and significant $(p=.000)$. Furthermore, we found support for the hypothesized positive effect of perceived preference fit on sharing intention $(\mathrm{p}=.017)$ and the positive effect of perceived enjoyment on sharing intention $(\mathrm{p}=.021)$. Accordingly, $\mathrm{H} 4 \mathrm{a}$ and $\mathrm{H} 4 \mathrm{~b}$ can be confirmed.

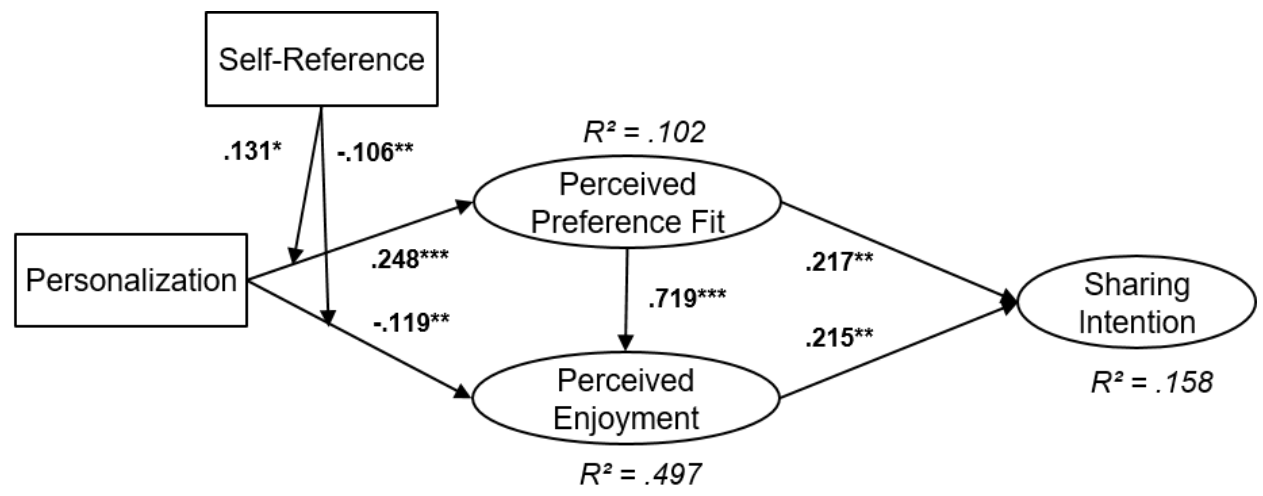

Figure 3. PLS estimation results $\left(n=194 ;{ }^{* * *} p<0.01 ;{ }^{* *} p<0.05 ;{ }^{*} p<0.1\right)$ 


\section{Discussion}

In this study, we aimed to investigate how personalized and self-referential news offerings impact the sharing of news content. The results provide two main findings: First, by drawing on the S-O-R framework, the results show that personalization impacts users' cognitive and affective reactions toward the news offering, which in turn affect their sharing intention. Second, our findings reveal that self-reference influences the relationship between personalization and users' internal reaction.

Our results indicate that providing users with a personalized news offering is effective in increasing their perceived preference fit. The inherent nature of personalization systems to constantly match users' needs with the provided content [8] elicits an increased perception of preference fit among users. These insights are in line with findings from prior studies [10]. However, our study extends previous findings by highlighting the importance of providing users with self-referential cues, which activate concepts stored in users' self-schemes [13] and strengthen the positive effect of personalization on perceived preference fit. Hence, our findings empirically confirm the customized label effect proposed by Simonson [30], suggesting that self-referential labels enhance the perceived fit of personalized news offerings.

In contrast to the findings of prior studies [10], our results do not support the assumption that personalization increases users' perceived enjoyment. However, a mediation analysis revealed that the insignificant total effect is due to a negative direct effect and a positive indirect effect via perceived preference fit. The negative direct effect of personalization on perceived enjoyment could be explained by the fact that users perceive a personalized news offering as less diverse than an offering that addresses a large variety of topics, which allows users to discover new areas of interest. This is in line with previous findings indicating that expected variety heightens the perception that an activity is interesting and enjoyable [41]. Our results revealed that the negative direct effect of personalization on perceived enjoyment can be mitigated by providing users with self-referential cues. Self-referential cues, resulting in more extensive information processing [18], may help users understand that the limited content diversity is due thereto that the offering is tailored to their personal preferences. This, in turn, decreases the negative direct effect of personalization on perceived enjoyment.

Furthermore, we find that users experience a news offering as more enjoyable if they notice that the offering matches their preferences. Previous research has shown that technological systems which provide users with information that fits the task they want to fulfil, increase the enjoyment derived from interacting with a system [31]. Accordingly, perceiving a greater overlap between the presented articles and own interests facilitates the selection of interesting news content and, therefore, increases the perceived enjoyment.

In accordance with the confirmation bias theory [22], our results show that users are more likely to share news content that reflects their current interests and preferences. Building on the theory of homophily [24], we argue that users tend to associate with peers who are similar to themselves and thus often recommend news content addressing common preferences as the recommended content might be perceived as relevant by their peers. Our findings also yield new insights on filter bubbles [42] in the news media. Prior research [43] found empirical evidence that personalization systems drive the formation of filter bubbles, i.e. restricted content diversity and partial information blindness, resulting in ideological polarization in today's media landscape. We find that personalization systems further foster the dissemination of content that matches own preferences, thus leading to an expansion of filter bubbles among like-minded peers.

Regarding users' affective intervening process, we find that news content is more likely to be shared when it elicits positive emotional reactions. The more enjoyment users derive from the news offering, the more likely they are to share the news articles. This is in line with findings from Berger and Milkman [3] who investigated the sharing of news articles on the 'New York Times' website and revealed that positive news content was more viral. Likewise, Stieglitz and DangXuan [4] found that emotionally charged Twitter messages tend to be disseminated more often and more quickly than those that elicit no emotions.

\section{Theoretical and practical implications}

Our findings offer theoretical and practical implications. From a theoretical perspective, our study contributes to existing literature by combining insights from information systems, marketing, and communication research. Previous research on the dissemination of online news mainly focused on the impact of content, network, and user characteristics on news sharing. To the best of our knowledge, our study is the first to examine the impact of personalization and self-referential cues on users' sharing intention. Specifically, our findings provide empirical support for the two opposing effects of personalized news content on users' internal reaction: On the one hand, personalization of the news offering reduces information overload by enabling increased preference fit. On the other hand, it diminishes the enjoyment 
derived from the offering which might be explained by reduced content diversity. However, this negative direct effect can be mitigated by the enjoyment users derive from the preference fit of the personalized news offering. Accordingly, personalization ultimately promotes news sharing driven by the tendency to share content that supports the users' current beliefs. In line with the phenomenon of homophily, we find that personalized news offerings not only influence which content users consume but also which content they share. Hence, personalization systems foster the dissemination of similar content among like-minded peers. This finding contributes to the current debate about the impact of personalization systems on the formation of filter bubbles [42] and echo chambers [23]. Second, we showed that the incorporation of selfreferential cues improves users' reaction toward the personalized news offering. In line with prior research on transparency features of personalization systems [26], this indicates that informational cues enhance the understanding and acceptance of personalization systems. Overall, our study enhances the understanding of how personalized news offerings impact the intention to share news articles.

Likewise, our findings provide valuable practical insights for news providers. Personalizing the news offering is likely to stimulate the sharing of news articles. This effect can be enhanced by integrating selfreferential cues such as the headline "your personalized news feed", in the news offering since self-reference enhances the impression that a news offering actually matches own preferences. However, to prevent the formation and proliferation of filter bubbles, news providers should not only exploit their knowledge about users' preferences but also actively promote other topics and opinions to ensure rich content diversity.

\section{Limitations and future research}

There are several limitations to our study, which open various avenues for further research. First, our sample consists mainly of students, which might limit the representativeness of the results. Although the use of student samples is common practice in consumer research, replicating this study with different samples may increase the generalizability of the findings. Second, our experimental design was based on a rather simple personalization procedure. Since we relied solely on explicit user information to personalize the news offering, future research should incorporate more advanced personalization algorithms using implicit user data. As implicit personalization is less likely to diminish news content diversity than explicit personalization [13], this could yield further insights into the relationship between personalization and perceived enjoyment. Third, our study focuses on how users' internal processing, i.e. perceived enjoyment and perceived preference fit, explains the impact of personalization on news sharing. Since previous studies have shown the importance of user characteristics in the context of news sharing [e.g. 20, 21], future research should expand our research model by incorporating user characteristics, such as self-serving, altruistic and social motives, as moderating variables.

In summary, our proposed model provides a promising foundation for further investigations of the impact of personalization on users' sharing intention. Therefore, we aim to conduct a large-scale field experiment to validate and further advance our understanding of the proposed relationships.

\section{References}

[1] Newman, N., Fletcher, R., Kalogeropoulos, A., and Nielsen, R., "Reuters Institute Digital News Report 2019", Oxford, 2019, available at http://www.digitalnewsreport.org/survey/.

[2] Kümpel, A.S., Karnowski, V., and Keyling, T., "News Sharing in Social Media: A Review of Current Research on News Sharing Users, Content, and Networks", Social Media + Society, 1(2), 2015, pp. 1-14.

[3] Berger, J., and Milkman, K.L., "What Makes Online Content Viral?", Journal of Marketing Research, 49(2), 2012, pp. 192-205.

[4] Stieglitz, S., and Dang-Xuan, L., "Emotions and Information Diffusion in Social Media-Sentiment of Microblogs and Sharing Behavior", Journal of Management Information Systems, 29(4), 2013, pp. 217-248.

[5] Susarla, A., Oh, J.-H., and Tan, Y., "Influentials, Imitables, or Susceptibles? Virality and Word-of-Mouth Conversations in Online Social Networks", Journal of Management Information Systems, 33(1), 2016, pp. 139-170.

[6] Yoo, E., Gu, B., and Rabinovich, E., "Diffusion on Social Media Platforms: A Point Process Model for Interaction among Similar Content", Journal of Management Information Systems, 36(4), 2019, pp. 1105-1141.

[7] Lee, C.S., and Ma, L., "News Sharing in Social Media: The Effect of Gratifications and Prior Experience", Computers in Human Behavior, 28, 2012, pp. 331-339.

[8] Adomavicius, G., and Tuzhilin, A., "Personalization Technologies: A Process-Oriented Perspective", Communications of the ACM, 48(10), 2005, pp. 83-90.

[9] Herington, C., and Weaven, S., "E-Retailing by Banks: EService Quality and Its Importance to Customer Satisfaction", European Journal of Marketing, 43(9/10), 2009, pp. 12201231.

[10] Benlian, A., "Web Personalization Cues and Their Differential Effects on User Assessments of Website Value", Journal of Management Information Systems, 32(1), 2015, pp. 225-260.

[11] Berger, B., Matt, C., Steininger, D.M., and Hess, T., "It Is Not Just About Competition with "Free": Differences 
between Content Formats in Consumer Preferences and Willingness to Pay", Journal of Management Information Systems, 32(3), 2015, pp. 105-128.

[12] Eppler, M.J., and Mengis, J., "The Concept of Information Overload - a Review of Literature from Organization Science, Accounting, Marketing, MIS, and Related Disciplines (2004)", in Meckel, M., and Schmid, B.F. (Eds.), Kommunikationsmanagement Im Wandel, Gabler, Wiesbaden, 2008, pp. 271-305.

[13] Tam, K.Y., and Ho, S.Y., "Understanding the Impact of Web Personalization on User Information Processing and Decision Outcomes", MIS Quarterly, 30(4), 2006, pp. 865890.

[14] Li, C., "When Does Web-Based Personalization Really Work? The Distinction between Actual Personalization and Perceived Personalization", Computers in Human Behavior, 54, 2016, pp. 25-33.

[15] Kramer, T., "The Effect of Measurement Task Transparency on Preference Construction and Evaluations of Personalized Recommendations", Journal of Marketing Research, 44(2), 2007, pp. 224-233.

[16] Choi, J., Lee, H.J., and Kim, H.-W., "Examining the Effects of Personalized App Recommender Systems on Purchase Intention: A Self and Social-Interaction Perspective", Journal of Electronic Commerce Research, 18(1), 2017, pp. 73-102.

[17] Kircher, T.T., Senior, C., Phillips, M.L., Benson, P.J., Bullmore, E.T., Brammer, M., Simmons, A., Williams, S.C., Bartels, M., and David, A.S., "Towards a Functional Neuroanatomy of Self Processing: Effects of Faces and Words", Cognitive Brain Research, 10(1-2), 2000, pp. 133144.

[18] Rogers, T.B., Kuiper, N.A., and Kirker, W.S., "SelfReference and the Encoding of Personal Information", Journal of Personality and Social Psychology, 35(9), 1977, pp. $677-$ 688.

[19] Klein, S.B., and Loftus, J., "The Nature of Self-Referent Encoding: The Contributions of Elaborative and Organizational Processes", Journal of Personality and Social Psychology, 55(1), 1988, pp. 5-11.

[20] Berger, J., "Word of Mouth and Interpersonal Communication: A Review and Directions for Future Research", Journal of Consumer Psychology, 24(4), 2014, pp. 586-607.

[21] Holton, A.E., Baek, K., Coddington, M., and Yaschur, C., "Seeking and Sharing: Motivations for Linking on Twitter", Communication Research Reports, 31(1), 2014, pp. 33-40.

[22] Geva, H., Oestreicher-Singer, G., and Saar-Tsechansky, M., "Using Retweets When Shaping Our Online Persona: Topic Modeling Approach", MIS Quarterly, 43(2), 2019, pp. 501-524.

[23] Quattrociocchi, W., Scala, A., and Sunstein, C.R., "Echo Chambers on Facebook", SSRN, 2016, available at https://ssrn.com/abstract=2795110.

[24] Bapna, R., and Umyarov, A., "Do Your Online Friends Make You Pay? A Randomized Field Experiment on Peer Influence in Online Social Networks", Management Science, 61(8), 2015, pp. 1902-1920.

[25] Mehrabian, A., and Russell, J.A., An Approach to Environmental Psychology, The MIT Press, Cambridge, MA, 1974.
[26] Xu, J., Benbasat, I., and Cenfetelli, R.T., "The Nature and Consequences of Trade-Off Transparency in the Context of Recommendation Agents", MIS Quarterly, 38(2), 2014, pp. 379-406.

[27] Jacoby, J., "Stimulus-Organism-Response Reconsidered: An Evolutionary Step in Modeling (Consumer) Behavior", Journal of Consumer Psychology, 12(1), 2002, pp. 51-57.

[28] Koufaris, M., "Applying the Technology Acceptance Model and Flow Theory to Online Consumer Behavior", Information Systems Research, 13(2), 2002, pp. 205-223.

[29] Jiang, Z., and Benbasat, I., "Research NoteInvestigating the Influence of the Functional Mechanisms of Online Product Presentations", Information Systems Research, 18(4), 2007, pp. 454-470.

[30] Simonson, I., "Determinants of Customers' Responses to Customized Offers: Conceptual Framework and Research Propositions", Journal of Marketing, 69(1), 2005, pp. 32-45.

[31] Parboteeah, D.V., Valacich, J.S., and Wells, J.D., "The Influence of Website Characteristics on a Consumer's Urge to Buy Impulsively", Information Systems Research, 20(1), 2009, pp. 60-78.

[32] Shaw, J.C., Wild, E., and Colquitt, J.A., "To Justify or Excuse?: A Meta-Analytic Review of the Effects of Explanations", Journal of Applied Psychology, 88(3), 2003, pp. 444-458.

[33] Li, T., and Unger, T., "Willing to Pay for Quality Personalization? Trade-Off between Quality and Privacy", European Journal of Information Systems, 21(6), 2012, pp. 621-642.

[34] The New York Time, accessed June 10, 2020, https://www.nytimes.com/.

[35] BBC News, accessed June 10, 2020, https://www.bbc.com/news/world.

[36] Reichheld, F.F., "The One Number You Need to Grow", Harvard Business Review, 81(12), 2003, pp. 46-55.

[37] Zhu, M., Billeter, D.M., and Inman, J.J., "The DoubleEdged Sword of Signaling Effectiveness: When Salient Cues Curb Postpurchase Consumption", Journal of Marketing Research, 49(1), 2012, pp. 26-38.

[38] Ringle, C.M., Wende, S., and Becker, J.-M., "SmartPLS 3": Boenningstedt: SmartPLS GmbH, available on http://www.smartpls.com.

[39] Hair, J.F., Hult, G.T.M., Ringle, C., and Sarstedt, M., A Primer on Partial Least Squares Structural Equation Modeling (PLS-SEM), Sage Publications, 2, Thousand Oaks, CA, 2016. [40] Zhao, X., Lynch Jr, J.G., and Chen, Q., "Reconsidering Baron and Kenny: Myths and Truths About Mediation Analysis", Journal of Consumer Research, 37(2), 2010, pp. 197-206.

[41] Dimmock, J., Jackson, B., Podlog, L., and Magaraggia, C., "The Effect of Variety Expectations on Interest, Enjoyment, and Locus of Causality in Exercise", Motivation and Emotion, 37(1), 2013, pp. 146-153.

[42] Pariser, E., The Filter Bubble: What the Internet Is Hiding from You, Penguin, New York, NY, 2011.

[43] Dylko, I., Dolgov, I., Hoffman, W., Eckhart, N., Molina, M., and Aaziz, O., "The Dark Side of Technology: An Experimental Investigation of the Influence of Customizability Technology on Online Political Selective Exposure", Computers in Human Behavior, 73, 2017, pp. 181190. 